\title{
On Optimal Coordinated Search Technique to Find a Randomly Located Target
}

\author{
Ajab A. Alfreedi ${ }^{1}$, Mohamed Abd Allah El-Hadidy ${ }^{1,2, *}$ \\ ${ }^{1}$ Mathematics and Statistics Department, College of Science, Taibah University, Yanbu, Saudi Arabia \\ 2 Mathematics Department, Faculty of Science, Tanta University, Tanta, Egypt
}

\begin{abstract}
We present the cooperation between two searchers start at the origin to seek for the pipeline hole. The pipeline searched with random distances and velocities through the time by each searcher. There is no available information about the target position to the searchers all the time. We study this problem in the case of bounded asymmetric and unbounded symmetric hole distribution. Rather than finding the expected value of the time detection, we find the optimal search plan which minimizes this detection time. The effectiveness of this model is illustrated using a numerical example.
\end{abstract}

Keywords Search theory, Coordinated search technique, Symmetric distribution.

AMS 2010 subject classifications 37A50, 60K30, 90B40.

DOI: $10.19139 /$ soic-2310-5070-724

\section{Introduction}

The Linear search problem for a lost target either located or moved is often a time-critical issue, when the target is very important, e.g., searching for a bomb in the street. The prime focus is to find and search for the cast ways in the smallest possible amount of the time. When the target is located somewhere on the real line, according to a known probability distribution, the searcher searches for it with a know velocity and tries to find it in minimal expected time. It is assumed that the searcher can change the direction of its motion without any loss of time. The target can be detected only, if the searcher reaches the target. In an earlier work, this problem has been studied extensively in many variations, mostly by Beck et al. [1, 2, 3, 4, 5, 6, 7], Franck [8] and Reyniers [9, 10].

The main contribution in the case of a randomly moving target is showing the existence of finite and optimal search plan when the searcher starts its motion from the origin or any point rather than the origin. Also, obtaining the optimal search plan which minimizes the expected value of the first meeting time between the searcher and the target. For example, when the target moves with a random walk, Mc Cabe[11], Mohamed [12], Mohamed and El-Hadidy [13] and El-Hadidy and Abou Gabal[17] presented the conditions which give the existence of finite and optimal search plan. In some earlier works, a linear search method has been presented to find a Brownian target motion such as El-Rayes et al.[14], Mohamed et al.[15] and El-Hadidy [23]. They also discussed the finiteness of finite and an optimal search plan. A comprehensive discussion of many aspects of search problem is found in El-Hadidy et al.[18, 19, 27, 21, 22, 23, 24, 25, 26, 27, 28, 30]. Finding the holes in the existing oil pipeline in the depths of the seas and oceans help to the lack of a disaster such as that occurred in the Gulf of Mexico in 2010 and led to the death of fish. One of the famous search methods, is the coordinated linear search method which

\footnotetext{
*Correspondence to: Mohamed Abd Allah El-Hadidy, Mathematics Department, Faculty of Science, Tanta University, Tanta, Egypt (Email:
} melhadidi@science.tanta.edu.eg).

ISSN 2310-5070 (online) ISSN 2311-004X (print)

Copyright (C) 2019 International Academic Press 
considers two unit-speed searchers starting at the origin point seek a stationary target hidden according to a know bounded symmetric and asymmetric distribution. The objective is to minimize the expected time of the searchers to return to the origin after one of them has found the target as in Reyniers [9, 10]. There are two constraints in the coordinated linear search method: (1) the speed of the searchers is equal to one; (2) the searchers return to the origin point and one of them is waiting for the other. We ask ourselves what happen if the straight line is flat like the street which contains the tops and bottoms (i.e., non smoothing)?. This necessarily leads to change the searcher speed through the time. In addition, the objective of the coordinated search is to minimize the expected search time, How? Where the constrain (2) is found. Here, we let $X$ be a position of the hole which is randomly in the pipeline and has asymmetric distribution. We have two sensors $S_{1}$ and $S_{2}$ starting together and looking for the hole from the point $a_{0}=b_{0}=0$ on the pipeline. We will address the shortcoming of the previous works by considering the velocities of the searchers are random through the time. The sensor $S_{1}$ searches the right part of the pipeline and $S_{2}$ searches the left part as in figure 2 with random velocity $v$. In order to overcome the second constraint, the searchers will use the modern communication technology to relate to each other and not returning to the origin point. Thus, there is no stand by and return time. Therefore, the expected value of the detection time is less than the half value of the expected time that given in Reyniers $[9,10]$. We use the audio signals as a communication method under the water instead of electromagnetic waves, because the electromagnetic waves do not spread well in salt water, see Website https://www.youtube.com/watch?v=evIwfBCru4I. Our aim is to find the expected value of the time for the sensors to detect the hole. Also, we wish to obtain the optimal search plan to find the hole in minimum time. This paper is organized as follows: Section 2 discusses the assumptions which modeling the search problem for bounded asymmetric hole distribution. Beside that we determine the optimal search strategy that minimizes the expected value of the time for detecting this hole. Also in this Section 2 we give an example with numerical results that can show the effectiveness of this model. In Section 3 we study the problem which discussed in Section 2 when the hole has an arbitrary unbounded symmetric distribution. Finally, the paper concludes with a discussion of the results and directions for the future research.

\section{Problem formulation}

The problem under study can be formally described as follows.

\subsection{The searching framework}

The space of search: The search space is a real line $L$.

The target: The target is randomly located on $L$ with known distribution.

The means of search: We will consider that searching the target will be carried out by two searchers $S_{1}$ and $S_{2}$. The searcher $S_{1}$ will search the right part of the line and $S_{2}$ will search the other part.

\subsection{The searching technique for bounded asymmetric distribution hole}

The searching process of all searchers will start at the same moment. For example $S_{1}$ will do its searching process in the right part as follows:

I. Start at the origin and go to the $+v e$ part of $L$ as far as $a_{1}=c_{1}-c_{0}$ such that $0<a_{1} \leq H_{1}^{2}$. Then, using the communication device (which works with audio signals (see in Figure 1) to send a report to the origin (the ship has a radar received an audio signal) whether the hole pipe is detected or not. He waits the response from the ship to complete the searching process or not. If the reply came to not complete the searching process, then the hole has been detected by the other searcher. Otherwise, go to step II.

II. Since, the hole has not detected, then $S_{1}$ will go to the $+v e$ part of $L$ as far as $a_{2}=c_{2}-c_{1}$. Then, $S_{1}$ send the report to the ship. And so on until the hole will detect (see Figure 2)

Zaburdaev et al. [31] show that the random velocity of the particle has a probability density function: $h(v)=$ $\left[\delta\left(v-v_{0}\right)+\delta\left(v+v_{0}\right)\right] / 2,-\infty \leq v \leq \infty$, where $v_{0}$ is the initial velocity and $\delta$ is Dirac Delta function. Here, we 


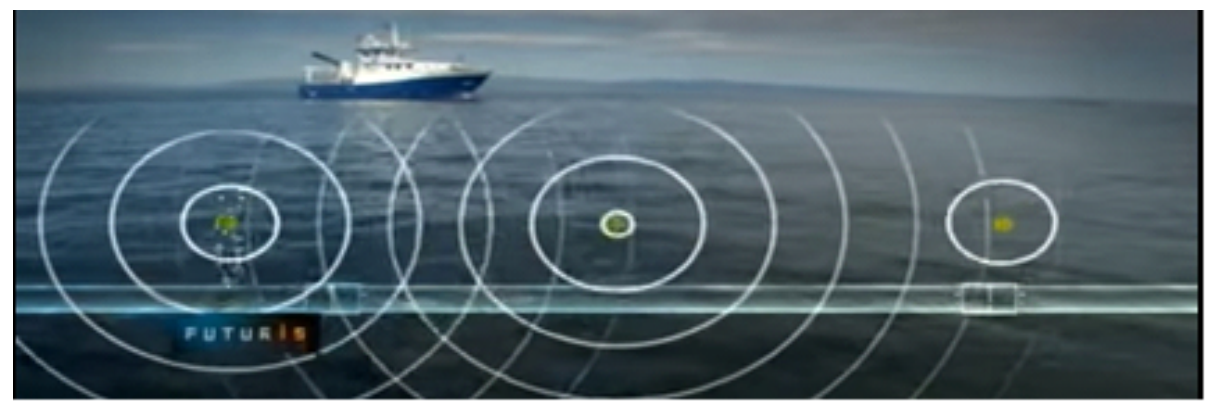

Figure 1. Ship has a radar received an audio signals.

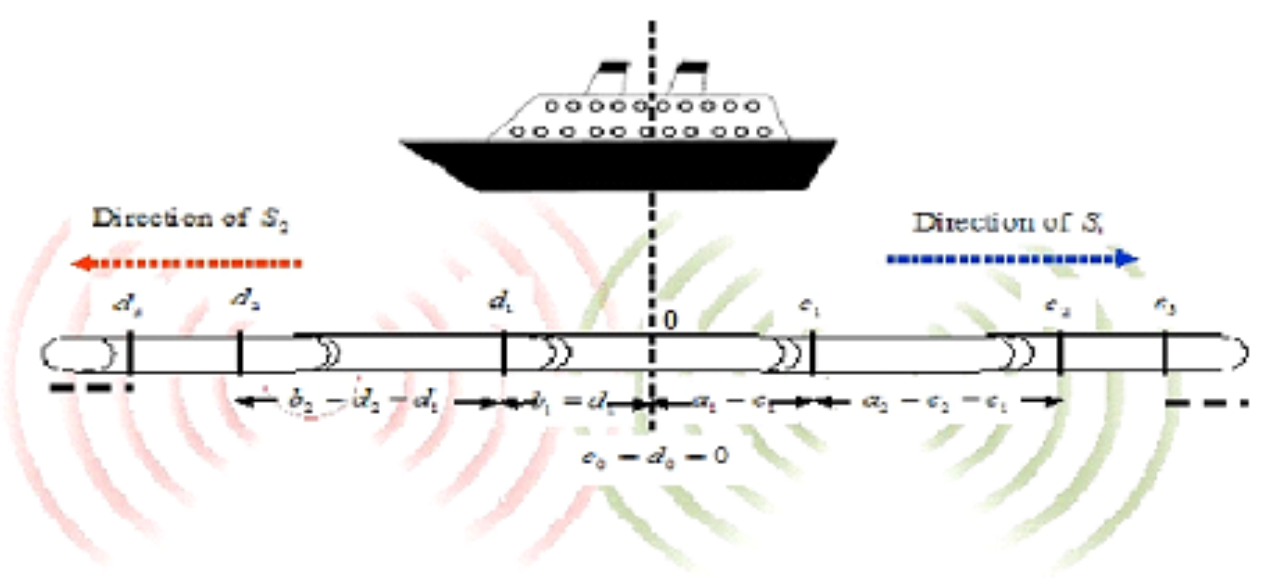

Figure 2. Cooperative coordinated search technique for finding a pipeline hole.

consider any searcher has a random velocity with probability density function (PDF):

$$
g(v)=\left|v-v_{0}\right| \delta\left(v^{2}-v_{0}\right),-\infty \leq v \leq \infty
$$

In the optimal case, we consider the length of the maximum distance which traveled by $S_{1}$ before the $i^{\text {th }}$ dialing in the right part be $H_{i}$ (in the left part be $\tilde{H}_{i}$ ). The nature of the searching process for the pipe hole make the distances which traveled by the searchers are random and given by $0<a_{i} \leq H_{i}^{2}$ in the right part where $a_{i}=c_{i}-c_{i-1}$ (and $0<b_{i} \leq \tilde{H}_{i}^{2}$ in the left part where $b_{i}=d_{i}-d_{i-1}$ ). We consider the PDF of the random distance is given by:

$$
f_{1}\left(a_{i}\right)=\frac{1}{H_{i} \sqrt{a}_{i}}-\frac{1}{H_{i}^{2}}, 0<a_{i} \leq H_{i}^{2}
$$

And,

$$
f_{2}\left(b_{i}\right)=\frac{1}{\tilde{H}_{i} \sqrt{b}_{i}}-\frac{1}{\tilde{H}_{i}^{2}}, 0<b_{i} \leq \tilde{H}_{i}^{2}
$$

In this work, the searchers wish to minimize the expected time to detect the pipe hole. Let $X$ be a random variable that represents the position of the pipe hole. In addition, this hole has a certain asymmetric distribution on the pipe. 
It means every searcher moves on the pipe with different distances according to the probability of the existence of the hole on the pipe. Thus, we can assume that $H_{i}<\tilde{H}_{i}$ (or $H_{i}>\tilde{H}_{i}$ ). Consequently, if their exist $\Gamma \geq 0$ is a random variable has a known distribution with expected value $E(\Gamma)$, then we can consider $\tilde{H}_{i}=H_{i}+E(\Gamma)$. Hence, (3) become

$$
f_{2}\left(b_{i}\right)=\frac{1}{\left(H_{i}+E(\Gamma)\right) \sqrt{b}_{i}}-\frac{1}{\left(H_{i}+E(\Gamma)\right)^{2}}, 0<b_{i} \leq\left(H_{i}+E(\Gamma)\right)^{2}
$$

The searchers $S_{1}$ and $S_{2}$ follow search paths $a$ and $b$, respectively, to detect the hole. The search path $a$ of $S_{1}$ is completely defined by a sequence $\left\{a_{i} ; i \geq 0\right\}, i$ is a nonnegative integer. Also, the search path $b$ of $S_{2}$ is completely defined by a sequence $\left\{b_{i} ; i \geq 0\right\}$. Let the search plan be defined by $\phi=(a, b) \in \Phi$ where $\Phi$ is the set of all search plans. Define,

$$
\alpha=\inf \{x ; F(x)>0\} \text { and } \beta=\sup \{x ; F(x)<1\}
$$

where $F(x)$ is the distribution function of the hole position, $\alpha$ is the minimum value of $b_{i}$ and $\beta$ is the maximum value of $a_{i}$.

Let $\gamma$ be a measure of probability induced by the hole position and $\gamma(x, y)=F(y)-F(x)$. Also, let $\Omega(\phi)$ be the detection time by one of the searchers.

Theorem 1. The expected value of the detection time for the asymmetric hole distribution on the pipe is given by:

$$
E(\Omega(\phi))=\frac{1}{6} \sum_{i=0}^{\infty}\left(H_{i+1}^{2}\left[\gamma\left(\alpha, d_{i}\right)\right]-\left(H_{i+1}+E(\Gamma)\right)^{2}\left[\gamma\left(c_{i}, \beta\right)\right]\right) .
$$

Proof. Since, each searcher moves on the pipe with different and random distances (have PDFs (2) and (4) in the two parts of the pipe) according the probability of the existence of the hole on the pipe. Thus, the expected value of the distance in the right is,

$$
E\left(a_{i}\right)=\int_{0}^{H_{i}^{2}} a_{i}\left(\frac{1}{H_{i} \sqrt{a}_{i}}-\frac{1}{H_{i}^{2}}\right) d a_{i}=\frac{H_{i}^{2}}{6}
$$

and in the left part is,

$$
E\left(b_{i}\right)=\int_{0}^{\left(H_{i}+E(\Gamma)\right)^{2}} b_{i}\left(\frac{1}{\left(H_{i}+E(\Gamma)\right) \sqrt{b}_{i}}-\frac{1}{\left(H_{i}+E(\Gamma)\right)^{2}}\right) d b_{i}=\frac{\left(H_{i}+E(\Gamma)\right)^{2}}{6} .
$$

Also, the expected value of the velocity is given by $E(v)= \pm 1$. Consequently, the expected value of detection time in the right part is $\Omega_{1}=\frac{E\left(a_{i}\right)}{+1}=\frac{H_{i}^{2}}{6}$ (in the left part is $\Omega_{2}=\frac{E\left(b_{i}\right)}{-1}=-\frac{\left(H_{i}+E(\Gamma)\right)^{2}}{6}$ ). If the hole lies in $\left.] a_{0}, a_{1}\right]$, then $\Omega_{2}=-\frac{\left(H_{1}+E(\Gamma)\right)^{2}}{6}$. And, if the hole lies in $\left.] a_{1}, a_{2}\right]$, then $\Omega_{2}=-\left[\frac{\left(H_{1}+E(\Gamma)\right)^{2}+\left(H_{2}+E(\Gamma)\right)^{2}}{6}\right]$. Thus, if the hole lies in $\left.] a_{k-1}, a_{k}\right]$, then $\Omega_{2}=-\left[\frac{\left(H_{1}+E(\Gamma)\right)^{2}+\left(H_{2}+E(\Gamma)\right)^{2}+\ldots+\left(H_{k}+E(\Gamma)\right)^{2}}{6}\right]$. Similarly, the hole lies in $\left[b_{k}, b_{k-1}[\right.$, then $\Omega_{1}=\left[\frac{H_{1}^{2}+H_{2}^{2}+\ldots+H_{k}^{2}}{6}\right]$ and so on. According to the assumptions that the hole has a certain asymmetric distribution on the pipe, we get the expected value of the detection time as follows:

$$
\begin{aligned}
& E(\Omega(\phi))=-\left[\frac{\left(H_{1}+E(\Gamma)\right)^{2}}{6}\right]\left[\gamma\left(c_{0}, c_{1}\right)\right]-\left[\frac{\left(H_{1}+E(\Gamma)\right)^{2}+\left(H_{2}+E(\Gamma)\right)^{2}}{6}\right]\left[\gamma\left(c_{1}, c_{2}\right)\right]-\ldots \\
& - \\
& \quad\left[\frac{\left(H_{1}+E(\Gamma)\right)^{2}+\left(H_{2}+E(\Gamma)\right)^{2}+\ldots+\left(H_{k}+E(\Gamma)\right)^{2}}{6}\right]\left[\gamma\left(c_{k-1}, c_{k}\right)\right]-\ldots \\
& \quad+\frac{H_{1}^{2}}{6}\left[\gamma\left(d_{1}, d_{0}\right)\right]+\left[\frac{H_{1}^{2}+H_{2}^{2}}{6}\right]\left[\gamma\left(d_{2}, d_{1}\right)\right]+\ldots+\left[\frac{H_{1}^{2}+H_{2}^{2}+\ldots+H_{k}^{2}}{6}\right]\left[\gamma\left(d_{k}, d_{k-1}\right)\right]+\ldots \\
& =\frac{H_{1}^{2}}{6}\left[\gamma\left(d_{1}, d_{0}\right)+\gamma\left(d_{2}, d_{1}\right)+\ldots+\gamma\left(d_{k}, d_{k-1}\right)+\ldots\right]+\frac{H_{2}^{2}}{6}\left[\gamma\left(d_{2}, d_{1}\right)+\ldots+\gamma\left(d_{k}, d_{k-1}\right)+\ldots\right]+\ldots \\
& + \\
& +\frac{H_{k}^{2}}{6}\left[\gamma\left(d_{k}, d_{k-1}\right)+\ldots\right]+\ldots \\
& -
\end{aligned}
$$




$$
\begin{aligned}
& -\frac{\left(H_{2}+E(\Gamma)\right)^{2}}{6}\left[\gamma\left(c_{1}, c_{2}\right)+\ldots+\gamma\left(c_{k-1}, c_{k}\right)+\ldots\right]-\ldots \\
& -\frac{\left(H_{k}+E(\Gamma)\right)^{2}}{6}\left[\gamma\left(c_{k-1}, c_{k}\right)+\ldots\right]-. . \\
& =\frac{H_{1}^{2}}{6}\left[\gamma\left(\alpha, d_{0}\right)\right]+\frac{H_{2}^{2}}{6}\left[\gamma\left(\alpha, d_{1}\right)\right]+\ldots+\frac{H_{k}^{2}}{6}\left[\gamma\left(\alpha, d_{k-1}\right)\right]+\ldots \\
& -\frac{\left(H_{1}+E(\Gamma)\right)^{2}}{6}\left[\gamma\left(c_{0}, \beta\right)\right]-\frac{\left(H_{2}+E(\Gamma)\right)^{2}}{6}\left[\gamma\left(c_{1}, \beta\right)\right]-\ldots-\frac{\left(H_{k}+E(\Gamma)\right)^{2}}{6}\left[\gamma\left(c_{k-1}, \beta\right)\right]-. . \\
& =\frac{1}{6} \sum_{i=0}^{\infty}\left(H_{i+1}^{2}\left[\gamma\left(\alpha, d_{i}\right)\right]-\left(H_{i+1}+E(\Gamma)\right)^{2}\left[\gamma\left(c_{i}, \beta\right)\right]\right) .
\end{aligned}
$$

Since, $0<c_{i}-c_{i-1} \leq H_{i}^{2}$ then if their exist $\varepsilon \geq 0$ one can get $H_{i}^{2}=\left(c_{i}-c_{i-1}+\varepsilon\right)^{2}$. Hence, (6) take the form:

$$
E(\Omega(\phi))=\frac{1}{6} \sum_{i=0}^{\infty}\left(\left(c_{i+1}-c_{i}+\varepsilon\right)^{2}\left[\gamma\left(\alpha, d_{i}\right)\right]-\left(c_{i+1}-c_{i}+\varepsilon+E(\Gamma)\right)^{2}\left[\gamma\left(c_{i}, \beta\right)\right]\right) .
$$

Also, if their exist $\xi \geq 0$ one can get $\tilde{H}_{i}^{2}=\left(d_{i}-d_{i-1}+\xi\right)^{2}$. Compensate for $H_{i}=\tilde{H}_{i}-E(\Gamma)=d_{i}-d_{i-1}+\xi-$ $E(\Gamma)$ in (6) we get:

$$
E(\Omega(\phi))=\frac{1}{6} \sum_{i=0}^{\infty}\left(\left(d_{i+1}-d_{i}+\xi-E(\Gamma)\right)^{2}\left[\gamma\left(\alpha, d_{i}\right)\right]-\left(d_{i+1}-d_{i}+\xi\right)^{2}\left[\gamma\left(c_{i}, \beta\right)\right]\right) .
$$

Since, the hole has bounded asymmetric distribution, then we do not need to find the necessary and sufficient conditions that show the existence of optimal search plan. Immediately, we can get the optimal values of the points $c_{i}$ and $d_{i}$ which give the optimal path to detect the hole by using (7) and (8) as in the following section.

\subsection{Optimal search path for bounded asymmetric hole distribution}

Our aim is to minimize $E(\Omega(\phi))$. Then the main problem is to find the optimal value of $\left\{a_{i} ; i \geq 1\right\}$ and $\left\{b_{i} ; i \geq 1\right\}$ that give the optimal search path for a given hole distribution function from class $Q$. If such a search path exists, we call it optimal search path.

We deduce that, if $Q^{\prime}$ is a subclass of $Q$ for which only one element and if $a^{*}$ and $b^{*}$ are optimal values of $a$ and $b$; respectively, then the optimal search path will be in $Q^{\prime}$. As it can be seen that the search path depends on two unknown factors. Those are the target distribution $F(x)$, and the values of $a$ and $b$ used by the searchers. Since the values of $a$ and $b$ depend on the values of $\left\{c_{i} ; i \geq 0\right\}$ and $\left\{d_{i} ; i \geq 0\right\}$ then we need to find the optimal values $\left\{c_{i}^{*} ; i \geq 0\right\}$ and $\left\{d_{i}^{*} ; i \geq 0\right\}$. Let us assume, from now on, that the hole distribution be known. However, if we assume that the hole distribution $F(x)$ is also regular (i.e., the target distribution $F(x)$ is absolutely continuous with strictly positive density $f(x))$ and that $E(x)<\infty$.

Obtaining the optimal values $\left\{c_{i}^{*} ; i \geq 0\right\}$ is corresponding to solve the following Non-linear programming problem (NLP(1)):

NLP(1):

$$
\begin{array}{cl}
\min _{c_{i}} & \left(c_{i}-c_{i-1}+\varepsilon\right)^{2}\left[\gamma\left(\alpha, d_{i-1}\right)\right]-\left(c_{i}-c_{i-1}+\varepsilon+E(\Gamma)\right)^{2}\left[\gamma\left(c_{i-1}, \beta\right)\right] \\
& +\left(c_{i+1}-c_{i}+\varepsilon\right)^{2}\left[\gamma\left(\alpha, d_{i}\right)\right]-\left(c_{i+1}-c_{i}+\varepsilon+E(\Gamma)\right)^{2}\left[\gamma\left(c_{i}, \beta\right)\right],
\end{array},
$$

Sub. to: $\frac{c_{i}-c_{i-1}+\varepsilon}{c_{i}-c_{i-1}} \geq 1, \frac{c_{i+1}-c_{i}+\varepsilon}{c_{i+1}-c_{i}} \geq 1, c_{i}-c_{i-1}>0, c_{i+1}-c_{i}>0$,

$$
\begin{gathered}
\frac{c_{i}-c_{i-1}+\varepsilon+E(\Gamma)}{d_{i}-d_{i-1}} \geq 1, \frac{c_{i+1}-c_{i}+\varepsilon+E(\Gamma)}{d_{i+1}-d_{i}} \geq 1, d_{i}-d_{i-1}>0, \\
d_{i+1}-d_{i}>0, \varepsilon \geq 0, \Gamma \geq 0 .
\end{gathered}
$$

Definition 1 (Optimal solution): $c^{*} \in R$ are said to be an optimal solution of the above NLP, if there exist $c \in R$ such that $\Xi\left(c^{*}\right) \leq \Xi(c)$ for all $c \in R$.

However, on formulating the non-linear programming that closely describes and represents the real decision situation, various factors of the real system should be reflected in the description of the objective function and the constraints. Non-linear programming can be applied to various fields of study. It is widely used in business and economics, and is also utilized for some engineering problems. Industries that use non-linear programming 
models include transportation, energy, telecommunications, and manufacturing. It has proved useful in modeling diverse types of problems in planning, routing, scheduling, assignment, and design.

The above non-linear programming problem NLP(1) take the form:

NLP(2):

$$
\begin{array}{ll}
\min _{c_{i}} & \left(c_{i}-c_{i-1}+\varepsilon\right)^{2}\left[\gamma\left(\alpha, d_{i-1}\right)\right]-\left(c_{i}-c_{i-1}+\varepsilon+E(\Gamma)\right)^{2}\left[\gamma\left(c_{i-1}, \beta\right)\right] \\
& +\left(c_{i+1}-c_{i}+\varepsilon\right)^{2}\left[\gamma\left(\alpha, d_{i}\right)\right]-\left(c_{i+1}-c_{i}+\varepsilon+E(\Gamma)\right)^{2}\left[\gamma\left(c_{i}, \beta\right)\right]
\end{array}
$$

Sub. to : $1-\frac{c_{i}-c_{i-1}+\varepsilon}{c_{i}-c_{i-1}} \leq 0,1-\frac{c_{i+1}-c_{i}+\varepsilon}{c_{i+1}-c_{i}} \leq 0, c_{i-1}-c_{i}<0, c_{i}-c_{i+1}<0$,

$$
\begin{gathered}
1-\frac{c_{i}-c_{i-1}+\varepsilon+E(\Gamma)}{d_{i}-d_{i-1}} \leq 0,1-\frac{c_{i+1}-c_{i}+\varepsilon+E(\Gamma)}{d_{i+1}-d_{i}} \leq 0, d_{i-1}-d_{i}<0, \\
d_{i}-d_{i+1}<0,-\varepsilon \leq 0, \Gamma \leq 0 .
\end{gathered}
$$

From the Kuhn-Tucker conditions, we get

$$
\begin{aligned}
& 2\left(c_{i}-c_{i-1}+\varepsilon\right)\left[\gamma\left(\alpha, d_{i-1}\right)\right]-2\left(c_{i}-c_{i-1}+\varepsilon+E(\Gamma)\right)\left[\gamma\left(c_{i-1}, \beta\right)\right]-2\left(c_{i+1}-c_{i}+\varepsilon\right)\left[\gamma\left(\alpha, d_{i}\right)\right] \\
& +2\left(c_{i+1}-c_{i}+\varepsilon+E(\Gamma)\right)\left[\gamma\left(c_{i}, \beta\right)\right] \\
& +\left(c_{i+1}-c_{i}+\varepsilon+E(\Gamma)\right)^{2} f\left(c_{i}\right)+u_{1}\left(0-\frac{2\left(c_{i}-c_{i-1}+\varepsilon\right)\left(c_{i}-c_{i-1}\right)-\left(c_{i}-c_{i-1}+\varepsilon\right)}{\left(c_{i}-c_{i-1}\right)^{2}}\right)+u_{2}\left(0-\frac{2\left(c_{i+1}-c_{i}+\varepsilon\right)\left(c_{i+1}-c_{i}\right)-\left(c_{i+1}-c_{i}+\varepsilon\right)}{\left(c_{i+1}-c_{i}\right)^{2}}\right) \\
& +u_{3}\left(0-\frac{1}{d_{i}-d_{i-1}}\right)+u_{4}\left(0-\frac{1}{d_{i+1}-d_{i}}\right)+u_{5}(-1)+u_{6}(1)=0
\end{aligned}
$$

$$
\begin{gathered}
u_{1}\left(1-\frac{c_{i}-c_{i-1}+\varepsilon}{c_{i}-c_{i-1}}\right)=0, \\
u_{2}\left(1-\frac{c_{i+1}-c_{i}+\varepsilon}{c_{i+1}-c_{i}}\right)=0, \\
u_{3}\left(1-\frac{c_{i}-c_{i-1}+\varepsilon+E(\Gamma)}{d_{i}-d_{i-1}}\right)=0, \\
u_{4}\left(1-\frac{c_{i+1}-c_{i}+\varepsilon+E(\Gamma)}{d_{i+1}-d_{i}}\right)=0, \\
u_{5}\left(c_{i-1}-c_{i}\right)=0, \\
u_{6}\left(c_{i}-c_{i+1}\right)=0 .
\end{gathered}
$$

Many cases have been found to solve the above Eqs. (9)-(15). We found that the optimal values of $\left\{c_{i} ; i \geq 0\right\}$ are given only from the case: $u_{1}=u_{2}=\ldots=u_{6}=0$. Consequently, the optimal value of $c_{i+1}$ is given after solving the following equation,

$$
c_{i+1}^{2} f\left(c_{i}\right)+c_{i+1}\left[2 \gamma\left(\alpha, d_{i}\right)-2 \gamma\left(c_{i}, \beta\right)+2 f\left(c_{i}\right)\left(c_{i}+\varepsilon+E(\Gamma)\right)\right]=\Theta,
$$

where,

$$
\begin{aligned}
\Theta= & 2\left(c_{i}-c_{i-1}+\varepsilon\right)\left[\gamma\left(\alpha, d_{i-1}\right)\right]-2\left(c_{i}-c_{i-1}+\varepsilon+E(\Gamma)\right)\left[\gamma\left(c_{i-1}, \beta\right)\right]-2\left(-c_{i}+\varepsilon\right)\left[\gamma\left(\alpha, d_{i}\right)\right] \\
& +2\left(-c_{i}+\varepsilon+E(\Gamma)\right)\left[\gamma\left(c_{i}, \beta\right)\right]+\left(-c_{i}+\varepsilon+E(\Gamma)\right) f\left(c_{i}\right) .
\end{aligned}
$$

Also, the optimal values of $d_{i+1}$ is obtaining after solving the following NLP(3):

$$
\begin{array}{ll}
\min _{d_{i}} & \left(d_{i}-d_{i-1}+\xi-E(\Gamma)\right)^{2}\left[\gamma\left(\alpha, d_{i-1}\right)\right]-\left(d_{i}-d_{i-1}+\xi\right)^{2}\left[\gamma\left(c_{i-1}, \beta\right)\right] \\
& +\left(d_{i+1}-d_{i}+\xi-E(\Gamma)\right)^{2}\left[\gamma\left(\alpha, d_{i}\right)\right]-\left(d_{i+1}-d_{i}+\xi\right)^{2}\left[\gamma\left(c_{i}, \beta\right)\right]
\end{array}
$$


Sub. to : $1-\frac{d_{i}-d_{i-1}+\xi-E(\Gamma)}{d_{i}-d_{i-1}} \leq 0,1-\frac{d_{i+1}-d_{i}+\xi-E(\Gamma)}{d_{i+1}-d_{i}} \leq 0$,

$$
\begin{gathered}
1-\frac{d_{i}-d_{i-1}+\xi}{d_{i}-d_{i-1}} \leq 0,1-\frac{d_{i+1}-d_{i}+\xi}{d_{i+1}-d_{i}} \leq 0, \\
c_{i-1}-c_{i}<0, c_{i}-c_{i+1}<0, d_{i-1}-d_{i}<0, d_{i}-d_{i+1}<0,-\xi \leq 0, \Gamma \leq 0 .
\end{gathered}
$$

Applying Kuhn-Tucker conditions, we found that the optimal values of $d_{i+1}$ is given after solving the following equation,

$$
d_{i+1}^{2} f\left(d_{i}\right)+d_{i+1}\left[2 \gamma\left(c_{i}, \beta\right)-2 \gamma\left(\alpha, d_{i}\right)-2 f\left(d_{i}\right)\left(\xi-d_{i}-E(\Gamma)\right)\right]=\Lambda,
$$

where,

$$
\begin{aligned}
\Lambda= & 2\left(d_{i}-d_{i-1}+\xi-E(\Gamma)\right)\left[\gamma\left(\alpha, d_{i-1}\right)\right]-2\left(d_{i}-d_{i-1}+\xi\right)\left[\gamma\left(c_{i-1}, \beta\right)\right]+2\left(-d_{i-1}+\xi-E(\Gamma)\right)\left[\gamma\left(\alpha, d_{i}\right)\right] \\
& +\left(-d_{i}+\xi+E(\Gamma)\right)^{2} f\left(d_{i}\right)-2\left(\xi-d_{i}\right) \gamma\left(c_{i}, \beta\right) .
\end{aligned}
$$

\section{Example 1}

The oil is transferred to the consumer markets in both Europe and the United States of America during the Mediterranean through pipelines stretch for long distances. Supposing that there is a hole in the pipeline and its position $X$ has a double truncated standard normal distribution with mean 0 and variance 1 about the origin with probability density function:

$$
f(x)=\frac{1}{\operatorname{erf}\left(\frac{\beta}{\sqrt{2}}\right)-\operatorname{erf}\left(\frac{\alpha}{\sqrt{2}}\right)} \sqrt{\frac{2}{\pi}} e^{-\frac{x^{2}}{2}}, \quad \alpha \leq x \leq \beta
$$

and distribution function :

$$
F(x)=\left\{\begin{array}{lc}
\frac{\operatorname{erf}\left(\frac{\alpha}{\sqrt{2}}\right)-\operatorname{erf}\left(\frac{x}{\sqrt{2}}\right)}{\operatorname{erf}\left(\frac{\alpha}{\sqrt{2}}\right)-\operatorname{erf}\left(\frac{\beta}{\sqrt{2}}\right)}, & \alpha \leq x \leq \beta \\
0 & \text { Otherwise }
\end{array}\right.
$$

Also, let $\Gamma$ has an exponential distribution with parameter $\lambda>0$. Here, we consider that $1<\Gamma<4$, then $\Gamma$ has a truncated exponential distribution with mean $E(\Gamma)=\frac{(1+\lambda) e^{-\lambda}-(4 \lambda+1) e^{-4 \lambda}}{\lambda^{2}}$. In addition, let $\lambda=\varepsilon=1, \alpha=$ $-4, \beta=6, \xi=2$ and $c_{-1}=d_{-1}=c_{0}=d_{0}=0$. Based on the initial information, the distribution of the hole and using (16), $S_{1}$ should go to the +ve part of $L$ as far as 1.80253 unit distance. Simillary, in the the -ve part of $L$ and using (17), one can found that the other searcher should go -0.855632 unit distance. After substuting with $c_{1}=1.80253$ in (16), we found that $c_{2}=0.728099 i$ which is impossible. This means that $S_{1}$ was informed to stop the searching process and the hole was discovered by another searcher.

\section{Hole with an arbitrary unbounded symmetric distribution}

In this section we develop the theory which applies

In this section, we aim to find the hole which has an arbitrary unbounded symmetric distribution. Of course, the existence of a search plan with finite expected time could be taken for granted. We determine a condition on the hole distribution necessary and sufficient for a finite expected search time and then with the additional assumption of a decreasing continuous density function determine a necessary and sufficient condition for the existence of an optimal search strategy.

We begin by assuming that a hole is located on the pipeline according to a known unbounded distribution which is symmetric about zero in the sense that its cumulative distribution function $F$ satisfies $1-F(x)=F(-x)$. The set of search strategies available to the searchers is the set $C$ consisting of all strictly increasing sequences of positive numbers which tend to infinity. That is, $C=\left\{c=\left(c_{1}, c_{2}, \ldots\right): 0 \equiv c_{0}<c_{1}<c_{2}<\ldots, \lim _{i \rightarrow \infty} c_{i}=\infty\right\}$.

The searching process of all searchers will start at the same moment. The searcher $S_{1}$ will do its searching process in the right part who is following strategy $c$ goes from 0 to $c_{1}$ and tell the ship (origin), then to $c_{2}$ and so 
on. The 'left' searcher $S_{2}$ follows the path which is symmetric about zero to this path. If the hole lies in one of the intervals $\pm\left(c_{i-1}, c_{i}\right]$, which has probability $2\left(F\left(c_{i}\right)-F\left(c_{i-1}\right)\right)$, then the first connect with the ship (origin) after one of the searchers finds the hole occurs at time $\frac{1}{6} \sum_{\vartheta}^{i} H_{i \vartheta}^{2}=\frac{1}{6} \sum_{\vartheta}^{i}\left(c_{i \vartheta}-c_{(i-1) \vartheta}+\varepsilon\right)^{2}$.

Corollary 1. If the hole has a symmetric distribution about the origin, then the expected value of the time to detect it is given by:

$$
E(\Omega(\phi))=\frac{1}{3} \sum_{i=0}^{\infty}\left(\left(c_{i+1}-c_{i}+\varepsilon\right)^{2}\left[F\left(c_{i+1}\right)-F\left(c_{i}\right)\right),\right.
$$

The coordinated search technique for the oil pipeline hole is to determine the least expected time $E(\Omega(\phi))=\hat{E}(\Omega(\phi))$ to find this hole. The following recursion gives a necessary condition for a search plan to be optimal with respect to a given distribution.

Lemma 1. Let $F$ be a symmetric distribution with finite first absolute moment and density function $f$. Then, if $E(\Omega(\phi))=\hat{E}(\Omega(\phi))$ (i.e., if a is optimal where $a_{i}=c_{i}-c_{i-1}$ )it must satisfy the following recursion (where $\left.a_{-1}=c_{-1}=c_{0}=0\right)$ :

$$
c_{n+1}=\sqrt{\frac{2\left(c_{n}-c_{n-1}+\varepsilon\right)\left[F\left(c_{n-2}\right)-F\left(c_{n-1}\right)\right]}{f\left(c_{n}\right)}}+c_{n}-\varepsilon .
$$

Proof:

Whenever, the search path $a$ is optimal where $a_{i}=c_{i}-c_{i-1}$ (this leads to $\left.a_{i}+c_{i-1}=c_{i}\right)$ then $\left(c_{n-1}-\right.$ $\left.c_{n-2}, c_{n+1}-c_{n}\right) \rightarrow R$ defined by: $E\left(\Omega\left(c_{1}-c_{0}, \ldots, c_{n}-c_{n-1}, c_{n+1}-c_{n}, \ldots\right)\right)$ must have a global minimum. Since,

$$
\begin{aligned}
E(\Omega(\phi))= & \frac{1}{3} \sum_{i=0}^{\infty}\left(\left(c_{i+1}-c_{i}+\varepsilon\right)^{2}\left[F\left(c_{i+1}\right)-F\left(c_{i}\right)\right]\right) \\
= & \frac{1}{3} \sum_{i=0}^{\infty}\left(\left(a_{i+1}+\varepsilon\right)^{2}\left[F\left(c_{i-1}\right)-F\left(a_{i}+c_{i-1}\right)\right]\right) \\
= & \frac{1}{3}\left[\left(a_{1}+\varepsilon\right)^{2}\left[F\left(c_{-1}\right)-F\left(a_{0}+c_{-1}\right)\right]+\left(a_{2}+\varepsilon\right)^{2}\left[F\left(c_{0}\right)-F\left(a_{1}+c_{0}\right)\right]+\ldots\right. \\
& \left.\quad+\left(a_{n}+\varepsilon\right)^{2}\left[F\left(c_{n-2}\right)-F\left(a_{n-1}+c_{n-2}\right)\right]+\left(a_{n+1}+\varepsilon\right)^{2}\left[F\left(c_{n-1}\right)-F\left(a_{n}+c_{n-1}\right)\right]+\ldots\right] .
\end{aligned}
$$

Then, we have,

$$
\frac{\partial E(\Omega(\phi))}{\partial a_{n}}=2\left(a_{n}+\varepsilon\right)\left[F\left(c_{n-2}\right)-F\left(a_{n-1}+c_{n-2}\right)\right]+\left(a_{n+1}+\varepsilon\right)^{2}\left[-f\left(a_{n}+c_{n-1}\right)\right]=0,
$$

leads to,

$$
\left(a_{n+1}+\varepsilon\right)^{2}=\frac{2\left(a_{n}+\varepsilon\right)\left[F\left(c_{n-2}\right)-F\left(a_{n-1}+c_{n-2}\right)\right]}{f\left(a_{n}+c_{n-1}\right)} .
$$

Consequently,

$$
\left(c_{n+1}-c_{n}+\varepsilon\right)^{2}=\frac{2\left(c_{n}-c_{n-1}+\varepsilon\right)\left[F\left(c_{n-2}\right)-F\left(c_{n-1}\right)\right]}{f\left(c_{n}\right)} .
$$

Which equivalent to :

$c_{n+1}=\sqrt{\frac{2\left(c_{n}-c_{n-1}+\varepsilon\right)\left[F\left(c_{n-2}\right)-F\left(c_{n-1}\right)\right]}{f\left(c_{n}\right)}}+c_{n}-\varepsilon$.

We now consider the question of existence of an optimal strategy. The remainder of this section settles this question, at least for the important case of distributions given by a density which is continuous and decreasing for positive hole distances.

Theorem 2. Suppose that the hole at the position $x$ from the call center (origin) has an unbounded symmetric distribution $F$ which is given by density $f$. This density is continuous expect possibly at the origin. If $\lim _{x \rightarrow 0} f(x)=\infty$ then there is no optimal search strategy.

Proof: 
Let $w=\left(c_{1}-c_{0}, c_{2}-c_{1}, \ldots\right)$ be optimal for the hole distribution $F$ where $c_{1}-c_{0} \leq c_{2}-c_{1} \leq \ldots$ (according to the probability of the existence of the hole on the pipe). Also, let $c^{\prime}(x)$ be a search plan given by $\left(x-0, c_{1}-\right.$ $\left.c_{0}, c_{2}-c_{1}, \ldots\right)$. Eq. (20) can be written more simply as:

$$
E(\Omega(\phi))=\frac{1}{3} \sum_{i=0}^{\infty}\left(\left(c_{i+1}-c_{i}+\varepsilon\right)^{2}\left[1-F\left(c_{i}\right)\right) .\right.
$$

Define,

$$
\begin{aligned}
q(x) & =E\left(\Omega\left(w^{\prime}(x)\right)-E(\Omega(w(x))\right. \\
& =x(1-F(0))+\left(c_{1}-c_{0}+\varepsilon\right)(1-F(x))+\left(c_{2}-c_{1}+\varepsilon\right)\left(1-F\left(c_{1}\right)\right)+\ldots \\
& -\left(c_{1}-c_{0}+\varepsilon\right)\left(1-F\left(c_{0}\right)\right)-\left(c_{2}-c_{1}+\varepsilon\right)\left(1-F\left(c_{1}\right)\right)-\ldots \\
& =x(1-F(0))+\left(c_{1}-c_{0}+\varepsilon\right)(1-F(x))-\left(c_{1}-c_{0}+\varepsilon\right)\left(1-F\left(c_{0}\right)\right) \\
& =\frac{x}{2}+\left(c_{1}-c_{0}+\varepsilon\right)\left(\frac{1}{2}-F(x)\right) .
\end{aligned}
$$

If the hole at the call center then one can find that $q(0)=0$. Since, $x<c_{1}$ then if we let $0<\varpi<c_{1}$ with $q(\varpi)<0$, which means that the search plan $w^{\prime}(\varpi)$ has a lower expected than $c_{1}-c_{0}$, so that $w$ cannot be optimal.

\section{Conclusion and future work}

Coordinated search technique for a pipe hole has been presented, where the hole initial position is given by a random variable $X$. The searchers search the pipeline with random distances and velocities through the time. We formulate this problem when this hole has bounded asymmetric distribution. We obtain the expected value of the time for finding this hole as in Theorem 1. Moreover, we present the optimal search path that minimizes this expected value. We give an example with numerical results that can show the effectiveness of this model. In other hand we study this problem when the hole has an arbitrary unbounded symmetric distribution. It seems that the proposed model will be extendible to the multiple searchers case by considering the combinations of multiple holes.

\section{REFERENCES}

1. A. Beck, On the linear search problem, Israel J. Math., Vol. 2, no. 4, pp. 221-228, 1964.

2. A. Beck, More on the linear search problem, Israel J. Math., Vol. 3, no. 4, pp. 61-70, 1965.

3. A. Beck and M.Beck Son of the linear search problem, Israel J. Math., Vol. 48, no. (2-3), pp.109-122, 1984.

4. A. Beck and M.Beck The linear search problem rides again, Israel J. Math., Vol. 53, No. 3, pp. 365-372, 1986.

5. A. Beck and M.Beck The revenge of the linear search problem, SIAM J. Control Optim., Vol. 30, No. 1, pp.112-122, 1992.

6. A. Beck and D. Newman Yet more on the linear search problem, Israel J. Math., Vol. 8, No. 4, pp.419-429, 1970.

7. A. Beck and P. Warren The return of the linear search problem, Israel J. Math., Vol. 10, No. 2, pp.169-183, 1972.

8. W. Franck On an optimal search problem, SIAM Rev., Vol. 7, No. 4, pp.503-512, 1965.

9. D. J. Reyniers Coordinated search for an object on the line, Eur. J. Oper. Res., Vol. 95, No. 3, pp. 663-670, 1996.

10. D. J. Reyniers Coordinated two searchers for an object hidden on an interval, J. Oper. Res. Soc., Vol. 46, No. 11, pp. 1386-1392, 1995.

11. B. J. MC Cabe, Searching for one dimensional random walker, J. Appl. Prob., Vol. 11, No. 1, pp. 86-93,1974.

12. A.A. Mohamed, Generalized search for one dimensional random walker, Int. J. Pure Appl. Math., Vol. 19, No. 3, pp. 375-387, 2005.

13. A. Mohamed and M. E. El-Hadidy, Optimal multiplicative generalized linear search Plan for a discrete random walker, J. Optimization, Article ID 706176, 13 pages, http://dx.doi.org/10.1155/2013/706176,2013.

14. A. B. El-Rayes, A. A. Mohamed and H. M. Abou Gabal, Linear search for a brownian target motion, Acta Math. Scientia J., Vol 23 (B), No. 3, pp. 321-327, 2003.

15. A. Mohamed, M. Kassem and M. El-Hadidy, Multiplicative linear search for a brownian target motion, Appli. Mathematical Model., Vol. 35, No. 9, pp. 4127-4139, 2011.

16. M. El-Hadidy, Searching for a d-dimensional Brownian target with multiple sensors, Int. J. Mathematics in Operational Research, Vol. 9, No. 3, pp. 279-301, 2016.

17. M. El-Hadidy and H. Abou-Gabal, Coordinated Search for a Random Walk Target Motion, Fluctuation and Noise Letters, Fluctuation and Noise Letters, Vol. 17, No. 1, 1850002 (11 pages), 2018.

18. M. El-Hadidy, Optimal searching for a helix target motion, Sci. China Math., Vol. 58,No. 4, pp.749-762, 2015.

19. M. Kassem and M. El-Hadidy., Optimal multiplicative Bayesian search for a lost target, Appl. Math. Compu., Vol. 247, pp.795802,2014 
20. M. El-Hadidy, Optimal spiral search plan for a randomly located target in the plane, Int. J. of Operational Research, Vol. 22, No. 4, pp.454-465, 2015

21. M. El-Hadidy and A. H. El-Bagoury, Optimal search strategy for a three-dimensional randomly located target, Int. J. of Operational Research, in press [online] http://www.inderscience.com/info/ingeneral/forthcoming.php?jcode=ijor, 2015.

22. M. El-Hadidy and H. Abou-Gabal, Optimal searching for a randomly located target in a bounded known region, Int. J. of Computing Science and Mathematics, Vol. 6, No. 4, pp.392-403, 2015.

23. M. El-Hadidy, On Maximum Discounted Effort Reward Search Problem, Asia-Pacific Journal of Operational Research, Vol. 33, No. 3 (2016) 1650019 (30 pages), 2016.

24. M. El-Hadidy, Fuzzy Optimal Search Plan for N-Dimensional Randomly Moving Target, International Journal of Computational Methods, 13 (6)(2016) 1650038 (38 pages), 2016.

25. A. Mohamed, M. Kassem and M. El-Hadidy, M-states search problem for a lost target with multiple sensors, Int. J. Mathematics in Operational Research, Vol. 10, No. 1, pp. 104-135, 2017.

26. A. Mohamed, H. Abou-Gabal and M. El-Hadidy, Random search in a bounded area, Int. J. Mathematics in Operational Research, in press [online] http://www.inderscience.com/info/ingeneral/forthcoming.php?jcode=ijmor, 2015.

27. M. El-Hadidy, An optimal two-stages search plan for a random walk target motion in the plane, Int. J. Mathematics in Operational Research, in press [online]http://www.inderscience.com/info/ingeneral/forthcoming.php?jcode=ijmor, 2015.

28. M. El-Hadidy and M. Kassem, On minimum expected search time for a multiplicative random search problem, Int. J. of Operational Research', in press [online] http://www.inderscience.com/info/ingeneral/forthcoming.php?jcode=ijor, 2015.

29. A. Mohamed and M. El-Hadidy, Existence of a periodic search strategy for a parabolic spiral target motion in the plane, Afrika Matematika J., Vol. 24, No. 2, pp.145-160, 2013a.

30. M. El-Hadidy, Existence of finite parbolic spiral search plan for a Brownian target, Int. J. of Operational Research, in press [online] http://www.inderscience.com/info/ingeneral/forthcoming.php?jcode=ijor 2015.

31. V. Zaburdaev, M. Schmiedeberg and H. Stark, Random walks with random velocities PHYSICAL REVIEW E 78 (2008) 011119. 